\title{
Controversies of hepatectomy and adjuvant therapy for hepatocellular carcinoma: moving forward
}

\author{
Jian-Hong Zhong ${ }^{1,2}$, Bang-De Xiang ${ }^{1,2}$ \\ 'Department of Hepatobiliary Surgery, Affiliated Tumor Hospital of Guangxi Medical University, Nanning 530021, China. \\ ${ }^{2}$ Guangxi Liver Cancer Diagnosis and Treatment Engineering and Technology Research Center, Nanning 530021, China.
}

Correspondence to: Dr. Bang-De Xiang, Department of Hepatobiliary Surgery, Affiliated Tumor Hospital of Guangxi Medical University, He Di Rd. \#71, Nanning 530021, China. E-mail: xiangbangde@163.com

How to cite this article: Zhong JH, Xiang BD. Controversies of hepatectomy and adjuvant therapy for hepatocellular carcinoma: moving forward. Hepatoma Res 2019;5:18. http://dx.doi.org/10.20517/2394-5079.2019.01

Received: 2 Jan 2019 First Decision: 14 Jan 2019 Revised: 22 Mar 2019 Accepted: 23 Mar 2019 Published: 6 Jun 2019

Science Editor: Guang-Wen Cao Copy Editor: Cai-Hong Wang Production Editor: Huan-Liang Wu

Due to the prevelence of hepatitis B virus (HBV) and hepatitis C virus (HCV), occurrence of hepatocellular carcinoma (HCC) is increasing in many countries/regions, including China ${ }^{[1]}$. HBV and HCV infections, alcohol consumption, non-alcoholic steatohepatitis cirrhosis, or obesity pandemic are risk factors of HCC development. A recent survey study found about 70\% patients with HCC are diagnosed as intermediate or advanced disease because of the lack of significant syndrome in their early stage ${ }^{[2]}$. Main treatments of HCC include hepatectomy, liver transplantation, ablation (radiofrequency, microwave, cryoablation), transarterial chemoembolization, radiotherapy, chemotherapy, target therapy, and so on. Among these treatments, only hepatectomy, liver transplantation, and ablation are curative treatments, with a 70\% 5-year overall survival (OS) for early stage HCC. Hepatectomy is not recommended by Western official guidelines for intermediate and advanced stage HCC. However, Eastern official guidelines and many liver centres recommend hepatectomy for such patients who are with preserved liver function. Tumor recurrence, which occurs in $70 \%$ within 5 years after hepatectomy, is a major cause of death after hepatectomy ${ }^{[3]}$. This recurrence can be true recurrence relating to primary tumor (intrahepatic metastases), which occurs less than two years, or it can be due to the development of de novo tumors relating to liver disease (such as HBV/HCV and cirrhosis), which occurs at least two years later. Even so, none Western official guidelines recommend any effective adjuvant therapy to prevent HCC recurrence.

Therefore, there are at least three controversies in the field of HCC treatment between literature evidence and official guidelines. Namely:

1. Which HCC stage system is the best? Do we need more stage systems?

2. Should patients with intermediate or advanced stage HCC receive hepatectomy?

3. Should postoperative HCC patients receive adjuvant treatments?

cc) (i) $\odot$ The Author(s) 2019. Open Access This article is licensed under a Creative Commons Attribution 4.0 International License (https://creativecommons.org/licenses/by/4.0/), which permits unrestricted use, sharing, adaptation, distribution and reproduction in any medium or format, for any purpose, even commercially, as long as you give appropriate credit to the original author(s) and the source, provide a link to the Creative Commons license, and indicate if changes were made.

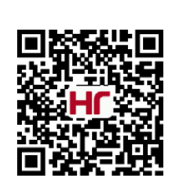


In recent two decades, nine systems have been proposed for staging HCC from Western to Eastern, including Cancer of the Liver Italian Program (CLIP) ${ }^{[4]}$, French Score ${ }^{[5]}$, Barcelona Clinic Liver Cancer (BCLC) staging ${ }^{[6]}$, the Model to Estimate Survival for HCC patients ${ }^{[7]}$, China liver cancer (2017 Edition) ${ }^{[8]}$, Chinese University Prognostic Index ${ }^{[9]}$, Hong Kong Liver Cancer (HKLC) system ${ }^{[10]}$, Japan Integrated Staging Score $^{[11]}$, and Italian Liver Cancer (ITA.LI.CA) system ${ }^{[8,12]}$. Among these stage systems, only the BCLC ${ }^{[6]}$, $\mathrm{HKLC}^{[10]}$, and China liver cancer $\left(2017\right.$ Edition $^{[8]}$ staging systems propose stage-appropriate treatment modalities. Even so, BCLC stage system is the only one endorsed by each version of the EASL ${ }^{[13]}$ and $\operatorname{AASLD}^{[14]}$.

However, each stage system has its own limitations. They leave large treatment gaps. For example, not each individual with HCC fall completely into his/her prespecified treatment modalities, and even those within the same HKLC or BCLC stage system may differ completely because of their different liver disease background. Many studies compared performance of different stage systems. Studies based on Western population found the BCLC system can predict overall survival and/or disease-free survival more accurately for Western patients with HCC than Eastern ones. However, studies based on Estern population found HKLC or China liver cancer (2017 Edition) staging system is better than Western ones ${ }^{[15,16]}$. Therefore, selection of stage system should be based on population characteristics.

Intermediate stage disease of BCLC system includes HCC involving asymptomatic multinodular tumors with a maximum diameter $>3 \mathrm{~cm}$ or $>3$ tumors without vascular invasion or extrahepatic spread. Earlier version of the BCLC system classified large solitary HCC beyong $5 \mathrm{~cm}$ with an expansive growth as intermediate disease. Namely, intermediate disease definition includes a wide range of patients according to liver function and tumour burden, which triggered a major controversy to further stratify intermediate stage HCC according to tumor burden and liver function ${ }^{[17-19]}$. Nowadays, guideline from European Association for the Study of the Liver ${ }^{[13]}$ and several reviews written by BCLC proponents seems trying to recalibrate their position stating that if technically feasible patients with large solitary HCC beyond $5 \mathrm{~cm}$ should be classified as BCLC stage A. Anyhow for patients with solitary HCC, hepatectomy is first-line treatment with good long-term OS.

Western official guidelines only recommend palliative treatments for intermediate disease, but not hepatectomy. Their recommendations did not completely reflect newest evidence by continuing to recommend transarterial chemoembolization, particularly in comparison with hepatectomy. The efficacy of transarterial chemoembolization is far from clear. Our systematic review involving large sample size with large solitary or multinodular HCC found median 1-, 3-, and 5-year OS after hepatectomy were $81 \%, 56 \%$, and $42 \%{ }^{[20]}$. For 4,945 patients with multinodular HCC, the corresponding OS were $75 \%, 48 \%$, and $30 \%{ }^{[21]}$. A recent large meta-analysis found significant OS benefits for hepatectomy over transarterial chemoembolization in BCLC stage B patients (hazard ratio, 0.59; 95\% confidence interval, 0.51-0.67; $P<$ $0.001)^{[22]}$. Nowadays, substantial evidence supported that hepatectomy would provide better OS than other palliative therapies, implying the possibility that some Western HCC guidelines are restricting many populations with intermediate stage HCC to palliative treatment. But actually, these populations could obtain more benefit from more aggressive hepatectomy.

In China, about half of HCC patients are diagnosed as HCC in an advanced stage ${ }^{[2]}$. Many studies compared the safety and efficacy of hepatectomy to transarterial chemoembolization ${ }^{[3,23,24]}$. Patients receiving either treatment modality showed similar safety. However, hepatectomy provided significantly longer median survival than transarterial chemoembolization, even after using propensity score analysis. Two recent large retrospective studies from Japan also found hepatectomy was associated with better OS for patients with portal vein tumor thrombus (PVTT) or hepatic vein thrombus ${ }^{[25,26]}$. The first study compared OS of 2,093 HCC patients with PVTT who underwent hepatectomy and 4,381 patients who received palliative 
therapies ${ }^{[25]}$. Patients in the hepatectomy group had significantly longer median OS than those received other treatments (2.87 years vs. 1.10 years. However, hepatectomy provided no OS benefit for those with PVTT affected the main trunk or contralateral branch (Vp3 or 4). Our systematic review involving 4,389 HCC patients with macrovascular invasion showed that hepatectomy provided median OS of 50\% at 1 year and $18 \%$ at 5 years ${ }^{[20]}$. However, median OS after sorafenib therapy was less than 1 year in presence of $\mathrm{PVTT}^{[27]}$. Moreover, radioembolization is associated with similar median OS with sorafenib in HCC patients with PVTT $^{[27]}$. These median survival time is not much higher than that of about 5 months after the best supportive care ${ }^{[13,28]}$. The OS benefit of palliative treatment is not obvious. Moreover, we should also consider that these treatments are always associated with risk of adverse events and high $\operatorname{costs}^{[29]}$.

These findings argue for expanding the Western officical liver guidelines ${ }^{[13,14]}$ to recognize hepatectomy as a therapeutic option for selected HCC patients with intermediate or advanced disease with good liver function (mainly Child-Pugh A). It may be true that some situations could decrease the efficacy and/or safety of hepatectomy. For instance, hepatectomy may be less effective and associated with more morbidity in HCC patients with multinodular tumors due to the possibility of microvascular invasion and liver/lung metastasis. In addition, liver cirrhosis and hepatitis activity may increase the risk of mortality, perioperative morbidity, and long-term tumor recurrence. However, continuous improvements in perioperative care and surgical technique support expanding the indications of hepatectomy. Surgeons and oncologists should not shy away from hepatectomy selection when it is feasible. At the same time, doctors should be fully conscious of the fact that the procedure is technically demanding ${ }^{[30]}$. This highlights the need to expand indications for hepatectomy.

But in fact, expanding indications of hepatectomy will translate into higher rate of tumor recurrence. Therefore, effective adjuvant therapy to prevent the recurrence of HCC is important to improve patients' long-term OS after hepatectomy. In recent decades, lots of studies have explored such therapies to prevent the recurrence of HCC, but until now, none has been officially recommended ${ }^{[13,14]}$.

Nowadays, many types of postoperative therapies to prevent HCC recurrence were reported, such as transarterial chemoembolization, nucleos(t)ide analogues (NAs), interferon- $\alpha$, adoptive immunotherapy, vitamin K2 analog, autologous tumor vaccination, sorafenib, capecitabine, and so on. Meta-analysis found a significant improvement in recurrence-free survival and OS when adjuvant transarterial chemoembolization is used for patients with high risk of early-phase recurrence, such as large tumor, vascular invasion, and multinodular tumors ${ }^{[31]}$. The other postoperative therapies with positive efficacy is NAs for patients with HBV-related $\mathrm{HCC}^{[32,33]}$, interferon- $\alpha$ for patients with HCV-infected $\mathrm{HCC}^{[34]}$. All these three therapies are with acceptable safety. However, the safety and efficacy of the following adjuvant therapies have not been definitively established, and need further clinical investigation: interferon- $\beta$ for patients with HCV-related HCC; interferon- $\alpha$ for patients with HBV-related HCC; vitamin K2 analog, autologous tumor vaccination, adoptive immunotherapy, heparanase inhibitor PI-88, iodine-131-labeled lipiodol, or capecitabine for patients with $\mathrm{HCC}^{[35,36]}$. In contrast, the following adjuvant therapies are not recommended for clinical use: tamoxifen, sorafenib, intravenous chemotherapy and systemic chemotherapy, octreotide, and branchedchain amino acid supplementation ${ }^{[37-39]}$. Though most of these reports can create a base for clinical use and further studies, their findings should be interpreted with caution due to their clinical heterogeneity among the trials (patients, liver disease, drugs, dosages, treatment duration, etc.) and their small sample size.

Early- and late-phase recurrence of HCC are associated with different risk factors, and patients will have different prognoses. Macrovascular invasion, tumor rupture, multinodular tumors, large tumor size, absence of a tumor capsule, poorly differentiated tumor, and narrow resection margin are associated with earlyphase recurrence. Liver cirrhosis, which is the risk factor of liver carcinogenesis, is associated with late-phase tumor recurrence. Moreover, HBV infection may contribute to both early- and late-phase recurrence. In 
China, nearly 90\% patients with HCC are infected with HBV. In addition, many of them have liver cirrhosis, show microvascular invasion or micrometastases before hepatectomy ${ }^{[38]}$. Therefore, almost each patient with HCC presents risk factors for early- and/or late-phase tumor recurrence. Adjuvant therapy for patients should be take into account the risk factors that they possess. Individuals presenting several such risk factors may benefit most from combination treatment modality dedicated to against both early- and late-phase tumor recurrence. However, few trials have investigated the safety and efficacy of combinated therapies. This content is urgently needed for further trials. They should think over the full profile of prognostic risk factors in included individuals so that ensure that individuals with similar risk factors are assigned the appropriate combination therapy.

In summary, official guidelines have been shown to be clinically useful for guiding research and treatment of $\mathrm{HCC}^{[13,14]}$. Nevertheless, despite the sometimes substantial evidence indicating the safety and efficacy of adjuvant therapies for specific patients, official guidelines do not recommend them as treatment options ${ }^{[13,14]}$. More and more worldwide studies suggest that (1) hepatectomy could be a suitable treatment for selected patients with intermediate or advanced stage HCC, as long as preserved liver function is adequate; and (2) adjuvant transarterial chemoembolization for individuals with high risk of early-phase recurrence, NAs for individuals with HBV-related HCC, and interferon- $\alpha$ for individuals with HCV-infected HCC, are associated with better OS. There is now room, rather than debating whether or not hepatectomy and adjuvant therapies should have a room for these individuals, for focusing better on selection criteria to further enhance the long-term benefits of hepatectomy and adjuvant therapies.

\section{DECLARATIONS}

\section{Authors' contributions}

Conceived the study: Xiang BD

Wrote and reviewed the manuscript: Zhong JH, Xiang BD

\section{Availability of data and materials}

Not applicable.

\section{Financial support and sponsorship}

This work was supported by the National Major Special Science and Technology Project (2017ZX10203207), Graduate Course Construction Project of Guangxi Medical University (YJSA2017014), the Foundation Ability Enhancement Project for Young Teachers in Guangxi Universities (2018KY0122), the Guangxi Natural Science Foundation (2018GXNSFBA138018), and Guangxi BaGui Young Scholars.

\section{Conflicts of interest}

Both authors declared that there are no conflicts of interest.

\section{Ethical approval and consent to participate}

Not applicable.

\section{Consent for publication}

Not applicable.

\section{Copyright}

(c) The Author(s) 2019.

\section{REFERENCES}

1. Bray F, Ferlay J, Soerjomataram I, Siegel RL, Torre LA, et al. Global cancer statistics 2018: GLOBOCAN estimates of incidence and mortality worldwide for 36 cancers in 185 countries. CA Cancer J Clin 2018;68:394-424. 
2. Zhong JH, Peng NF, You XM, Ma L, Xiang X, et al. Tumor stage and primary treatment of hepatocellular carcinoma at a large tertiary hospital in China: a real-world study. Oncotarget 2017;8:18296-302.

3. Zhong JH, Ke Y, Gong WF, Xiang BD, Ma L, et al. Hepatic resection associated with good survival for selected patients with intermediate and advanced-stage hepatocellular carcinoma. Ann Surg 2014;260:329-40.

4. A new prognostic system for hepatocellular carcinoma: a retrospective study of 435 patients: the Cancer of the Liver Italian Program (CLIP) investigators. Hepatology 1998;28:751-5.

5. Chevret S, Trinchet JC, Mathieu D, Rached AA, Beaugrand M, et al. A new prognostic classification for predicting survival in patients with hepatocellular carcinoma. Groupe d'Etude et de Traitement du Carcinome Hepatocellulaire. J Hepatol 1999;31:133-41.

6. Llovet JM, Bru C, Bruix J. Prognosis of hepatocellular carcinoma: the BCLC staging classification. Semin Liver Dis 1999;19:329-38.

7. Sang W, Chan SL, Chan AW. Proposal and validation of a new model to estimate survival for hepatocellular carcinoma patients. Eur J Cancer 2016;63:203-5.

8. Zhou J, Sun HC, Wang Z, Cong WM, Wang JH, et al. Guidelines for diagnosis and treatment of primary liver cancer in China (2017 Edition). Liver Cancer 2018;7:235-60.

9. Leung TW, Tang AM, Zee B, Lau WY, Lai PB, et al. Construction of the Chinese university prognostic index for hepatocellular carcinoma and comparison with the TNM staging system, the Okuda staging system, and the Cancer of the Liver Italian Program staging system: a study based on 926 patients. Cancer 2002;94:1760-9.

10. Yau T, Tang VY, Yao TJ, Fan ST, Lo CM, et al. Development of Hong Kong Liver Cancer staging system with treatment stratification for patients with hepatocellular carcinoma. Gastroenterology 2014;146:1691-700.

11. Kudo M, Chung H, Osaki Y. Prognostic staging system for hepatocellular carcinoma (CLIP score): its value and limitations, and a proposal for a new staging system, the Japan Integrated Staging score (JIS score). J Gastroenterol 2003;38:207-15.

12. Farinati F, Vitale A, Spolverato G, Pawlik TM, Huo TL, et al. Development and validation of a new prognostic system for patients with hepatocellular carcinoma. PLoS Med 2016;13:e1002006.

13. European Association for the Study of the Liver. EASL clinical practice guidelines: management of hepatocellular carcinoma. J Hepatol 2018;69:182-236.

14. Heimbach JK, Kulik LM, Finn RS, Sirlin CB, Abecassis MM, et al. AASLD guidelines for the treatment of hepatocellular carcinoma. Hepatology 2018;67:358-80.

15. Kolly P, Reeves H, Sangro B, Knopfli M, Candinas D, et al. Assessment of the Hong Kong Liver Cancer Staging System in Europe. Liver Int 2016;36:911-7.

16. Kim KM, Sinn DH, Jung SH, Gwak GY, Paik YH, et al. The recommended treatment algorithms of the BCLC and HKLC staging systems: does following these always improve survival rates for HCC patients? Liver Int 2016;36:1490-7.

17. Bolondi L, Burroughs A, Dufour JF, Galle PR, Mazzaferro V, et al. Heterogeneity of patients with intermediate (BCLC B) hepatocellular carcinoma: proposal for a subclassification to facilitate treatment decisions. Semin Liver Dis 2012;32:348-59.

18. Mo DC, Jia RR, Zhong JH. Hepatic resection compared to chemoembolization in intermediate to advanced-stage HCC: a comment for moving forward. Hepatology 2018. Epub ahead of print, DOI: 10.1002/hep.30409.

19. Wang YY, Zhong JH, Xu HF, Xu G, Wang LJ, et al. A modified staging of early and intermediate hepatocellular carcinoma based on single tumour $>7 \mathrm{~cm}$ and multiple tumours beyond up-to-seven criteria. Aliment Pharmacol Ther 2019;49:202-10.

20. Zhong JH, Rodriguez AC, Ke Y, Wang YY, Wang L, et al. Hepatic resection as a safe and effective treatment for hepatocellular carcinoma involving a single large tumor, multiple tumors, or macrovascular invasion. Medicine (Baltimore) 2015;94:e396.

21. Zhong JH, Wu FX, Li H. Hepatic resection associated with good survival for selected patients with multinodular hepatocellular carcinoma. Tumour Biol 2014;35:8355-8.

22. Hyun MH, Lee YS, Kim JH, Lee CU, Jung YK, et al. Hepatic resection compared to chemoembolization in intermediate- to advancedstage hepatocellular carcinoma: A meta-analysis of high-quality studies. Hepatology 2018;68:977-93.

23. Yuan BH, Yuan WP, Li RH, Xiang BD, Gong WF, et al. Propensity score-based comparison of hepatic resection and transarterial chemoembolization for patients with advanced hepatocellular carcinoma. Tumour Biol 2016;37:2435-41.

24. Peng ZW, Guo RP, Zhang YJ, Lin XJ, Chen MS, et al. Hepatic resection versus transcatheter arterial chemoembolization for the treatment of hepatocellular carcinoma with portal vein tumor thrombus. Cancer 2012;118:4725-36.

25. Kokudo T, Hasegawa K, Matsuyama Y, Takayama T, Izumi N, et al. Survival benefit of liver resection for hepatocellular carcinoma associated with portal vein invasion. J Hepatol 2016;65:938-43.

26. Kokudo T, Hasegawa K, Matsuyama Y, Takayama T, Izumi N, et al. Liver resection for hepatocellular carcinoma associated with hepatic vein invasion: a Japanese nationwide survey. Hepatology 2017;66:510-7.

27. de la Torre MA, Buades-Mateu J, de la Rosa PA, Lue A, Bustamante FJ, et al. A comparison of survival in patients with hepatocellular carcinoma and portal vein invasion treated by radioembolization or sorafenib. Liver Int 2016;36:1206-12.

28. Xiang X, Lau WY, Wu ZY, Zhao C, Ma YL, et al. Transarterial chemoembolization versus best supportive care for patients with hepatocellular carcinoma with portal vein tumor thrombus: a multicenter study. EJSO 2019. Available from: https://www.ejso.com/ article/S0748-7983(19)30387-7/abstract. [Last accessed on 23 Apr 2019]

29. Zhong JH, Torzilli G, Xing H, Li C, Han J, et al. Controversies and evidence of hepatic resection for hepatocellular carcinoma. BBA Clin 2016;6:125-30

30. Zhong JH, Ma L, Xiang BD, Li LQ, Yang T. We're still in an update process of the BCLC system. Ann Surg 2018;267:e23-e4.

31. Zhong JH, Li LQ. Postoperative adjuvant transarterial chemoembolization for participants with hepatocellular carcinoma: a metaanalysis. Hepatol Res 2010;40:943-53.

32. Huang G, Lau WY, Wang ZG, Pan ZY, Yuan SX, et al. Antiviral therapy improves postoperative survival in patients with hepatocellular carcinoma: a randomized controlled trial. Ann Surg 2015;261:56-66.

33. Huang G, Li PP, Lau WY, Pan ZY, Zhao LH, et al. Antiviral therapy reduces hepatocellular carcinoma recurrence in patients with low 
HBV-DNA levels: a randomized controlled trial. Ann Surg 2018;268:943-54.

34. Shen YC, Hsu C, Chen LT, Cheng CC, Hu FC, et al. Adjuvant interferon therapy after curative therapy for hepatocellular carcinoma (HCC): a meta-regression approach. J Hepatol 2010;52:889-94.

35. Mo HY, Liao YY, You XM, Cucchetti A, Yuan BH, et al. Timely meta-analysis on the efficacy of adoptive immunotherapy for hepatocellular carcinoma patients after curative therapy. PLoS One 2017;12:e0174222.

36. Zhong JH, Mo XS, Xiang BD, Yuan WP, Jiang JF, et al. Postoperative use of the chemopreventive vitamin K2 analog in patients with hepatocellular carcinoma. PLoS One 2013;8:e58082.

37. Zhong JH, Ma L, Li LQ. Postoperative therapy options for hepatocellular carcinoma. Scand J Gastroenterol 2014;49:649-61.

38. Zhong JH, Zhong QL, Li LQ, Li H. Adjuvant and chemopreventive therapies for resectable hepatocellular carcinoma: a literature review. Tumour Biol 2014;35:9459-68.

39. Bruix J, Takayama T, Mazzaferro V, Chau GY, Yang J, et al. Adjuvant sorafenib for hepatocellular carcinoma after resection or ablation (STORM): a phase 3, randomised, double-blind, placebo-controlled trial. Lancet Oncol 2015;16:1344-54. 